\title{
Orthodontic Treatment
}

National Cancer Institute

\section{Source}

National Cancer Institute. Orthodontic Treatment. NCI Thesaurus. Code C64248.

A dental treatment to correct malocclusions of the teeth and their surrounding structures. 